\title{
Belgeo
}

Revue belge de géographie

$1 \mid 2019$

Miscellaneous

\section{In Memoriam et bibliographie : Henri Nicolaï (1929 - 2019)}

\section{Christian Vandermotten}

\section{(2) OpenEdition}

1 Journals

Édition électronique

URL : http://journals.openedition.org/belgeo/36749

DOI : 10.4000/belgeo.36749

ISSN : 2294-9135

Éditeur :

National Committee of Geography of Belgium, Société Royale Belge de Géographie

Référence électronique

Christian Vandermotten, « In Memoriam et bibliographie : Henri Nicolaï (1929 - 2019)», Belgeo [En ligne], 1 | 2019, mis en ligne le 27 février 2020, consulté le 07 juillet 2020. URL : http:// journals.openedition.org/belgeo/36749; DOI : https://doi.org/10.4000/belgeo.36749

Ce document a été généré automatiquement le 7 juillet 2020

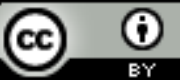

Belgeo est mis à disposition selon les termes de la licence Creative Commons Attribution 4.0 International. 


\section{In Memoriam et bibliographie : Henri Nicolaï (1929 - 2019)}

\section{Christian Vandermotten}

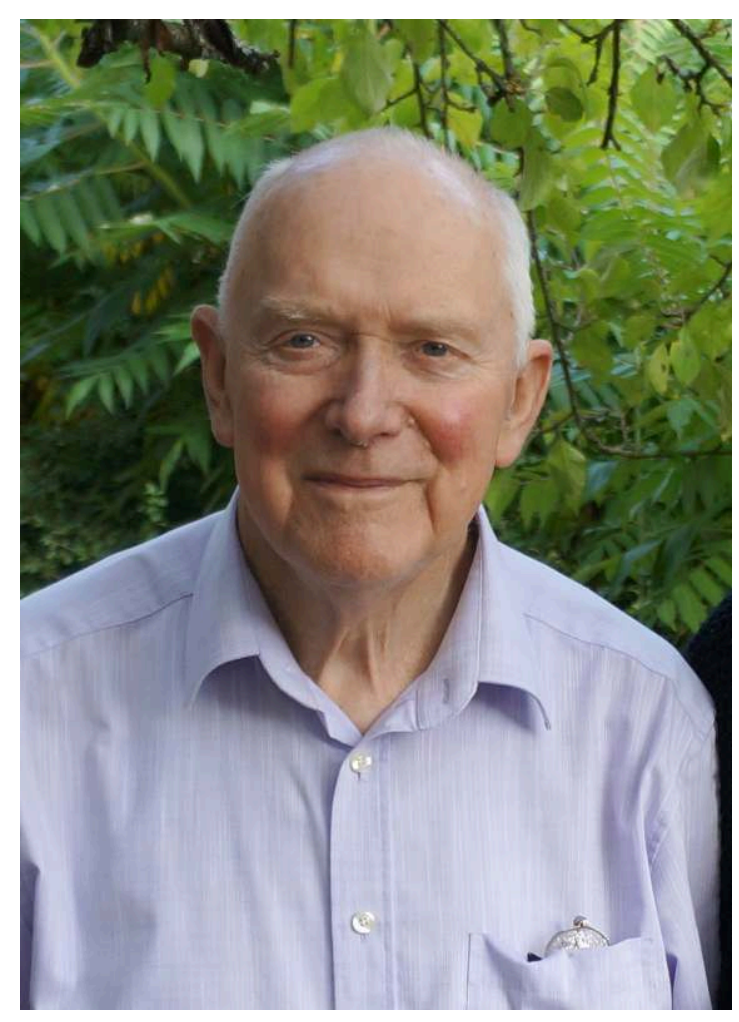

1 La Société Royale Belge de Géographie et le Comité National de Géographie ont chacun perdu un membre éminent et particulièrement dévoué. Henri Nicolaï s'est éteint le 30 novembre 2019. Nous ne le reverrons plus revenir régulièrement à la Société de Géographie, dans sa tenue soignée, s'enquérant des dernières nouvelles avec son habituelle discrétion, presque en s'excusant, fouillant dans les archives, dont il était le meilleur connaisseur, toujours prêt à référer avec une bienveillante autorité une proposition d'article sur l'Afrique soumise à Belgeo. 
2 Né à Verviers le 11 février 1929, Henri avait une sœur jumelle, Josette, qui deviendra elle aussi géographe. Ses parents ayant déménagé à Bruxelles, il y fit des études d'instituteur à l'École normale Charles Buls, qu'il termina en 1948, avant de passer le jury central et de s'inscrire à l'Université Libre de Bruxelles, où il obtint brillamment une licence en sciences géographiques en 1952.

3 C'est là que Pierre Gourou, dont il a sans doute été le plus fidèle disciple, lui a communiqué son goût pour l'étude de l'Afrique, qui ne s'est jamais démenti tout au long de sa carrière. Déjà élève-assistant à la fin de ses études de licence, Henri devint assistant en 1952, chef de travaux et chargé de cours en 1957, à la Faculté des sciences sociales, politiques et économiques de l'Université Libre de Bruxelles, professeur extraordinaire en 1961, avant même la défense de sa thèse de doctorat, consacrée au Kwilu, à l'Université de Bordeaux, où son maître Pierre Gourou avait lui-même enseigné pendant la guerre. Henri fut nommé professeur ordinaire à la Faculté des sciences de l'ULB en 1964, communiquant ses immenses savoirs, avec parfois une réserve excessive, non seulement aux géographes, mais aussi à de très nombreux groupes d'étudiants de la Faculté des sciences sociales, politiques et économiques, de la Faculté de philosophie et lettres ou de l'Institut d'Études européennes. Il fut co-directeur du Laboratoire de géographie humaine de l'Institut de géographie. Henri a toujours été très attentif à ses étudiants africains, en particulier à ceux qui firent leur doctorat sous sa direction minutieuse et auquels il continuait à s'intéresser avec attention et amitié après leur retour dans leur pays. Sa spécialisation d'africaniste - il fut sans doute un des plus fins connaisseurs de la géographie du Congo - n'excluait en rien une large curiosité scientifique et une vaste connaissance de très nombreux domaines de la géographie, et par exemple de la géographie de la Belgique et de la France, où l'on sentait une appropriation profonde des méthodes de la géographie régionale française classique, aujourd'hui largement oubliées par les jeunes géographes.

4 Henri avait effectué de nombreuses missions au Congo, dans le cadre des activités de la section de géographie du CEMUBAC, le Centre de Coopération médicale et scientifique de l'Université Libre de Bruxelles en Afrique centrale, en 1952, 1955, 1957, 1958 et 1959. Dès 1954, les premiers travaux africains d'Henri Nicolaï avaient été honorés, avec son collègue Jules Jacques, par le prix du concours annuel de l'Institut royal colonial belge. Le statut d'africaniste de grande qualité d'Henri a ensuite été honoré, tant en Belgique, où il était membre de l'Académie Royale des Sciences d'outre-mer, comme associé en 1980, puis comme membre titulaire en 1985, qu'en France, où il était depuis 2006 membre associé de l'Académie des Sciences d'outre-mer à Paris. Il a reçu en 1963 la médaille d'argent du prix Amaury d'Adhémar de la Société de Géographie de Paris. Il était membre de la Société des Africanistes et du comité belge de l'Association internationale des géographes et historiens de langue française. Henri était aussi un des piliers du Comité National de Géographie ainsi que de la Société Royale Belge de Géographie, qu'il a présidée de 1966 à 1969, de 1974 à 1977 et de 1994 à 1997. Il a aussi présidé la défunte Société Belge d'Études Géographiques. Il s'est largement investi durant les années 1990, avec Armando Montanari et d'autres, dans la mise sur pied d'EUGEO, l'association des sociétés de géographie européennes. Henri fut aussi membre du conseil d'administration du CEMUBAC.

5 Henri a vécu dans une famille très unie, avec son épouse, Marie-Thérèse Jacqmin - elle aussi géographe et qu'il a accompagnée ses deux dernières années dans la maison de repos où elle a dû être placée -, ses trois enfants, Jean-Philippe, Pierre et Damienne, et 
ses trois petits-enfants. Habitant Schaerbeek, il appréciait particulièrement sa résidence secondaire famennoise de La Loue, où il exerçait ses talents d'apiculteur.

Le souvenir d'Henri Nicolaï restera longtemps présent parmi les membres de la Société Royale Belge de Géographie et au Laboratoire de Géographie Humaine de l'Université Libre de Bruxelles. On trouvera ci-dessous sa bibliographie.

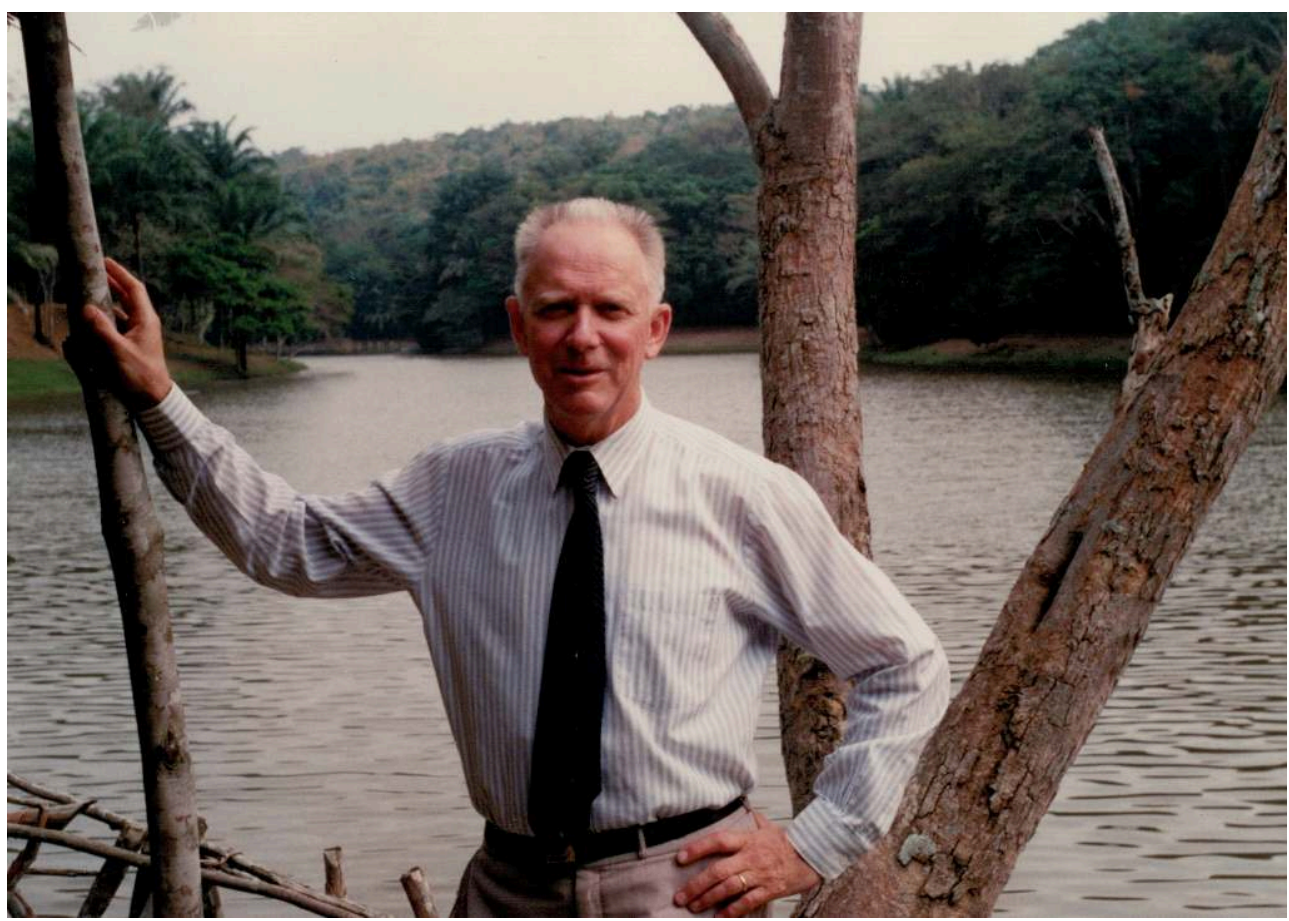

\section{Bibliographie d'Henri Nicolaï}

\section{Livres (comme auteur, co-auteur ou directeur) ou parties de livres}

1954

- (avec J. Jacques), La transformation des paysages congolais par le chemin de fer. L'exemple du B.C.K., Institut Royal Colonial Belge, Section des Sciences naturelles et médicales, mém. in- $8^{\circ}$, tome XXIV, fasc. 1, Bruxelles, $208 \mathrm{p}$.

1961

- Luozi. Géographie régionale d'un pays du Bas-Congo, Académie Royale des Sciences d'Outre-Mer, Classe des Sciences naturelles et médicales, mém. in- ${ }^{\circ}$, nouvelle série, tome XII, fasc. 5, Bruxelles, $95 \mathrm{p}$.

1963

- Le Kwilu. Etude géographique d'une région congolaise, CEMUBAC, Bruxelles, 423 p.

1969

- La Belgique. Le pays et les hommes, in Belgique : un panorama, Institut Belge d'Information et de Documentation, Bruxelles, pp. 11-51.

1976

- Géographie de l'Afrique Noire, in P. Salmon (éditeur), L'Afrique Noire. Histoire et culture, Meddens, Bruxelles, pp. 7-22.

1977

- Géographie de l'Afrique du Nord, in A. Destrée (éditeur), L'Afrique méditerranéenne. Unité et diversité, Meddens, Bruxelles, pp. 7-24. 
1996

- (avec P. Gourou et Mashini Dhi Mbita Mulenghe), L'espace zaïrois. Hommes et milieux. Progrès de la connaissance de 1949 à 1992, collection : Zaïre, Histoire et Société, Institut Africain, Bruxelles, L'Harmattan, Paris, 608 p.

1998

- La répartition et la densité de la population au Kivu, Académie royale des Sciences d'OutreMer, Classe des Sciences naturelles et médicales, mém. in- $8^{\circ}$, nouvelle série, tome 24 , fasc. 2, Bruxelles, 64 p., 2 cartes hors-texte en couleurs au 1/1 000000.

2000

- (avec P. Pélissier et J.P. Raison) (direction de), Un géographe dans son siècle. Actualité de Pierre Gourou, Karthala, Géotropiques, Collection "Hommes et sociétés”, Paris, 338 p. 2004

- (avec Vandermotten C. et Montanari A.) (guest editors), New trends and state of the geography in the European countries, Special issue for the 30th IGU congress, Glasgow, 15-20 August 2004, Belgeo, 1, 191 p.

2005

-L'image du Congo par la carte. De la boussole et de la planchette à l'avion, in La mémoire du Congo. Le temps colonial, Africa Tervuren (Musée royal de l'Afrique centrale), Snoeck, pp. 139-149.

2006

- (avec J.M. Decroly) (direction de), Mutations des territoires dans le Monde à l'aube du XXI siècle, Coll. Géotextes, L'Harmattan, Paris, 303 p.

2008

- (guest editor), Aspects of the History of the Belgian geography and cartography, Belgeo, 1, $154 \mathrm{p}$.

Articles, contributions à des colloques, des mélanges, des ouvrages encyclopédiques, cartes

\section{1}

- « La plaine de Montélimar et les pays d'alentour », Bulletin de la Société Belge d'Études Géographiques, 2, pp. 401- 426.

1952

- « Observations sur la population rurale de la Belgique », Bulletin de la Société Royale Belge de Géographie, pp. 59-78.

1953

- « Les couples stéréoscopiques, matériel didactique », La Géographie, pp. 20-25.

1956

- « Les problèmes du Kwango », Bulletin de la Société Belge d'Études Géographiques, pp. 247-275.

- "Fonctions démographiques des régions rurales », in La Mise en valeur des régions rurales, Fédération belge de l'Urbanisme et de l'Aménagement du Territoire, Congrès National, Bruxelles, pp. 1-6.

1957

- «La géographie économique des pays musulmans », Correspondance d'Orient, 1, Colloque sur le projet d'un marché commun afro-asiatique, Actes du 27-29 mai 1957, pp. 53-63. 
- « Le Bas-Kwilu et ses problèmes géographiques ", Bulletin de la Société Royale Belge de Géographie, pp. 21-66.

- « Disette à Kahemba. Une forme aiguë du problème kwangolais », Bulletin de la Société Belge d'Études Géographiques, pp. 311-320.

1959

- « Conflits entre groupes africains et décolonisation au Kasaï », Revue de l'Université de Bruxelles, octobre 1959- février 1960, pp. 131-144.

1964

- (avec P. Gourou), « Progrès de la connaissance géographique au Congo, au Rwanda et au Burundi en 1961, 1962 et 1963 », Bulletin de la Société Belge d'Études Géographiques, pp. 267-302.

- « Naissance d'une région en Afrique centrale. Le Kwilu », Bordeaux, Les Cahiers d'OutreMer, 67, pp. 292-313.

1965

- « Resuma geografica da Belgica », Panorama, Lisboa, dec., pp. 34-39.

1967

- «Quatre groupes humains du Congo méridional. Essai de géographie comparée » in Mélanges de géographie offerts à Omer Tulippe, pp. 571-583.

- « Divisions régionales et répartition de la population dans le sud-ouest du Congo ", Revue Belge de Géographie, pp. 161-227.

- « Progrès de la connaissance géographique au Congo, au Rwanda et au Burundi en 1964, 1965 et 1966 », Bulletin de la Société Belge d'Études Géographiques, pp. 273-315. 1969

- (avec H. Van der Haegen), « Industrie textile et métallurgique », Atlas de Belgique, planche 42.

- (avec E. Mai), « Congo », Westermann Lexikon der Geographie, 33 colonnes.

1970

- (avec Th. Brulard, F. Dussart et F. Snacken), « Divisions régionales de la Belgique », Atlas de Belgique, planche $50 \mathrm{~A}$.

- (avec Th. Brulard, F. Dussart et F. Snacken), « Exemples de régions géographiques », Atlas de Belgique, planches $51 \mathrm{~A}, \mathrm{~B}$ et $\mathrm{C}$.

- « Les modifications apportées par la croissance urbaine à l'organisation régionale ", in La croissance urbaine en Afrique Noire et à Madagascar, Colloques internationaux du C.N.R.S., Talence, pp. 219-229.

1971

- « Systèmes agricoles et développement au contact de la forêt et de la savane en Afrique subéquatoriale », Revue de Géographie de Montréal, XXV, 2, pp. 143-154.

1972

- « Les destinées d'un pays équatorial. Le lac Léopold II », in Études de géographie tropicale offertes à Pierre Gourou, École Pratique des Hautes Études, Sorbonne, VI ${ }^{\mathrm{e}}$ Section, Mouton, Paris, La Haye, pp. 357-370.

- « Zaïre », pp. 740-749, « Burundi », pp. 81-85, « Rwanda », pp. 461-465, in World Atlas of Agriculture, vol. Africa, Monographs edited by the Committee for the World Atlas of Agriculture.

1976

- « Géographie », in Liber Memorialis de l'Institut des Hautes Études de Belgique, Bruxelles, pp. IL-LV.

1978 
- (avec C. Vandermotten), « Aspects géographiques de la Mégalopole européenne », in Les mégalopoles dans l'Europe du Nord-Ouest, Colloque, Institut d'Études Européennes, Bruxelles, pp. 27-35.

1979

- «L'intervention coloniale dans l'agriculture villageoise », in Recherches de géographie rurale. Hommage au Professeur Frans Dussart, pp. 661-672.

1981

- (avec G. Lasserre), « Les systèmes de culture traditionnels et les phénomènes pionniers en Afrique tropicale ", in Les phénomènes de "frontière" dans les pays tropicaux, Travaux et Mémoires de l'Institut des Hautes Études de l'Amérique Latines, 34, Paris, pp. 95-115.

- « La géographie humaine africaine en Belgique », in Études Africaines en Europe. Bilan et inventaire, 1, A.C.C.T., Karthala, Paris, pp. 120-128.

- «Contributions belges aux études sur la démographie africaine », Ibid., pp. 129-132.

- «Contributions belges sur la sociologie rurale en Afrique », Ibid., p ; 133-134.

- «Contributions belges aux études sur la nutrition en Afrique », Ibid., p. 135-136.

- « Progrès de la connaissance géographique au Zaïre, au Rwanda et au Burundi de 1976 à 1980 », Bulletin de la Société Belge d'Études Géographiques, pp. 69-96.

1982

- «Présentation d'atlas de pays tropicaux francophones », Bulletin des Séances de l'Académie Royale des Sciences d'Outre-Mer, Bruxelles, 28, 3, pp. 339-351.

1983

- "Transition démographique et mobilité de la population en Afrique tropicale », Espace. Populations. Sociétés, Université de Lille, 1, pp. 61-66.

1986

- «Elisée Reclus et l'Afrique », Revue Belge de Géographie, 1, pp. 95-107.

- « Progrès de la connaissance géographique au Zaïre, au Rwanda et au Burundi de 1981 à 1985 », Bulletin de la Société Belge d'Études Géographiques, 2, pp. 175-223.

1987

- «Questions de géographie africaine », Revue Belge de Géographie, 3-4, pp. 101-103.

- «Réflexions sur la sous-urbanisation d'un espace africain, d'après le livre de P.

Sirven ", Revue Belge de Géographie, 3-4, pp. 141-148.

- « L'introduction à l'Histoire de l'Afrique" de Pierre Salmon, Revue Belge de Géographie,

3-4, pp. 209-211.

- «Originalités et tendances du semis urbain de l'Afrique tropicale, spécialement de

l'Afrique centrale ", in Recherches de géographie urbaine, Hommage au Professeur J.A. Sporck, Société Géographique de Liège, pp. 473-489.

1988

- «L'image de l'Afrique centrale au moment de la création de l'État Indépendant du Congo ", in Recueil d"études sur "Le centenaire de l'État Indépendant du Congo", Académie Royale des Sciences d'Outre-Mer, Bruxelles, pp. 13-39.

- « Répartition de la population et problèmes géographiques du Kivu d'altitude », in Actes du cinquantenaire du CEMUBAC, 23-24 mars 1988, Bruxelles, pp. 155-167.

1989

- «L'évolution du secteur tertiaire », in La Région de Bruxelles. Des villages d'autrefois à la ville d'aujourd'hui, Crédit Communal, Bruxelles, pp. 292-313. Idem en néerlandais : "Evolutie van de tertiaire sector", in Het Gewest Brussel. Van de oude dorpen tot de stad van nu, Gemeentekrediet, Brussel, pp. 292-313. 
- « Le citadin et son village. Un aspect de la relation ville-campagne au Zaïre », in Pauvreté et développement dans les pays tropicaux. Hommage au Professeur Guy Lasserre, Bordeaux, CEGET, CRET, Institut de Géographie de Bordeaux, 3, pp. 499-507.

- «Progrès de la connaissance géographique au Zaïre, au Rwanda et au Burundi en 1986, 1987 et 1988 », Bulletin de la Société Belge d'Études Géographiques, 1, pp. 159-207.

- « Enseignement et recherche sur la connaissance du milieu », in Symposium. L'Avenir des Sciences tropicales dans les Institutions universitaires belges (Milieu, Ressources, Santé), Bruxelles, 4 décembre 1987, Académie Royale des Sciences d'Outre-Mer, pp. 11-18. 1990

- « Fortes densités rurales de hautes terres africaines comparées à celles de l'Asie des Moussons ", in Regional Conference on Asian Pacific Countries, International Geographical Union, Beijing, Abstracts, volume 2, p. 14.

1991

- «Présentation du livre de P. Gourou, "L'Afrique noire. Nain ou géant agricole ? ” ", Bulletin des Séances de l'Académie royale des Sciences d'Outre-Mer, 37, 4, pp. 645-648. 1992

- « Géographie humaine et espace », in L'espace, Fondation Lucia De Brouckère pour la diffusion des sciences, Faculté des Sciences ULB, pp. 5-14.

- «Les mutations récentes des espaces africains », Bulletin des Séances de l'Académie royale des sciences d'Outre-Mer, 38, 4, pp. 563-578.

1993

- « Le Mouvement Géographique, un journal et un géographe au service de la colonisation du Congo ", in Mélanges Pierre Salmon, II, Histoire et ethnologie africaines, Civilisations, XLI, 1-2, pp. 257-277.

- «Progrès de la connaissance géographique du Zaïre, du Rwanda et du Burundi. Vingtième article. De 1989 à 1992 ", Bulletin de la Société Belge d'Études Géographiques, LXII, pp. 235-306.

1994

- « Les géographes belges et le Congo », in BRUNEAU Michel et DORY Daniel (directeurs), Géographies des colonisations. XV $V^{e}-X X^{e}$ siècles, coll. Géotextes, L'Harmattan, Paris, pp. 51-65.

- «Géographie et politique : Elisée Reclus et la création de l'Institut des Hautes Études, in Célébration du Centenaire de l'Institut des Hautes Études de Belgique, Colloque du jeudi 17 mars 1994, Institut des Hautes Études de Belgique, Bruxelles, pp. 17-26.

- « Réflexions sur la ville africaine comparée à la ville européenne », Acta Geographica Lovaniensis, 34, Liber Amicorum H. Van der Haegen, pp. 479-488.

1995

- «Les réseaux urbains de l'Afrique tropicale : origines, évolutions et tendances », in NZISABIRA Jean, Organisation de l'espace : le système dominant et son fonctionnement, Coll. Population et développement, 2, Academia-Bruylant, Louvain-la-Neuve, L'Harmattan, Paris, pp. 123-142.

- « Regional geography », in DENIS J. (éditeur), Geographical Research in Belgium, Comité National de Géographie, Bulletin de la Société belge d'Études géographiques, 64, 2, pp. 37-41. 1996

- «Les transformations d'un espace africain : le Kwilu », Bulletin de la Société Belge d'Études géographiques, 1, pp. 15-34. 
- «Réflexions sur les caractères originaux des paysages agraires de l'Afrique tropicale ", in Recherches de géographie humaine. Hommage au Professeur Charles Christians, Société Géographique de Liège, 1996, p. 311-319.

1997

- "Les géographes belges à la croisée des courants géographiques ", International Geographical Union, Union Géographique Internationale, Bulletin 47 (1), pp. 27-31.

- « Intervention à la commémoration du $175^{e}$ anniversaire de la Société de Géographie ", Acta Geographica, III, 111, pp. 40-45.

- «Composantes et implications démographiques du drame rwandais », in RwandaBurundi, Journée d'études, Bruxelles, I0 janvier 1997, Académie Royale des Sciences d'OutreMer, Bruxelles, pp. 11-33.

1998

- (avec P. Salmon), « Eau et santé dans le monde méditerranéen. Permanence de la question depuis l'antiquité gréco-romaine ", in L'eau : une valeur mondiale, Académie Royale des Sciences d'Outre-Mer, United Nations Brussels, Bruxelles, pp. 37-49.

- «Geografia, popolazione e violenza nelle regione dei Grandi Laghi », Terra d'Africa, VII, Edizioni Unicopoli, Milano, pp. 17-56.

- «In memoriam : Pierre Gourou (1900-1999)», Revue Belge de Géographie, 122, 2, pp. 109-118.

- «Bibliographie de Pierre Gourou », Revue Belge de Géographie, 122, 2, pp. 119- 130.

- « Réflexions sur un anniversaire. L'expédition de la Belgica 1887-1889 », Revue Belge de Géographie, 122, 3, pp. 197- 223.

1999

- « In Memoriam. Pierre GOUROU (1900-1999) », Bulletin de la Société Belge d'Études Géographiques, 1, pp. 7-13.

- « Pierre Gourou (1900-1999) », Les Cahiers d'Outre-Mer, Bordeaux, 208, pp. 341- 346. - (avec Maboloko Ngulambangu), « Frontière, diamant et pauvreté. Le cas de la frontière Congo-Angola au Kwango ", Revue Belge de Géographie, 4, pp. 255-275. 2000

- (avec Y. Verhasselt), « Health and tropical geography », Belgeo, 1-2-3-4 , pp. 103-113.

- « Poursuivre dans les traces de Pierre Gourou ? ", in NICOLAÏ H., PÉLISSIER P. et RAISON J.P. (sous la direction de), Un géographe dans son siècle. Actualité de Pierre Gourou, Karthala, Géotropiques, Paris, pp. 315- 319.

- «Pierre Gourou », Bulletin des Séances de l'Académie Royale des Sciences d'Outre-Mer, Bruxelles, 46, 4, pp. 531-540.

- (avec Ch. Kesteloot, I. Thomas, H. Beguin, W. De Lannoy, M. De Dapper, A. De Turck et A. Ozer), « Belgian geographers at work: the occupations of graduates in 1999 », BELGEO, Revue belge de Géographie, Special issue: 29th International Geographical Congress, 2000, p. 241-249.

2001

- «Le géographe et la décision politique ", in «Colloque à l'UNESCO - 26-27 juin 2000. La Géographie à l'aube du XXI ${ }^{\mathrm{e}}$ siècle : au service de la paix et d'un développement durable, respectueux de l'environnement et des cultures ", La Géographie, Acta Geographica, Paris, 173, hors série (n ${ }^{\circ} 1502$ bis), pp. 17-21.

- « La géographie », in HALLEUX R., VANDERSMISSEN J., DESPY-MEYER A., VANPAEMEL G., éditeurs scientifiques, Histoire des sciences en Belgique 1815-2000. Première partie : De la fondation des universités au Discours de Seraing, pp. 207-212 ; « Les sciences d'outre-mer. 
La géographie ", in idem, Deuxième partie, Les sciences d'outre-mer, pp. 247-250, Dexia, La Renaissance du Livre, Bruxelles.

- (avec P. Salmon), « Eau et santé dans le monde méditerranéen. Permanence de la question depuis l'Antiquité gréco-romaine ", in Thérapies, médecine et démographie antiques, Études réunies par J.-N. Corvisier, C.Didier, M.Valdher, Artois Presses Université, Collection "Histoire", pp. 13-24.(reprise du texte de 1998 publié par l'ARSOM).

2002

- (avec A. L. Jaumotte), « Notice sur Pierre Gourou », Annuaire 2002, Académie Royale de Belgique, Bruxelles, pp. 31-40.

- « Il contributo della geografia alle decisioni. Le potenzialità di EUGEO », Bolletino della Società Geografica Italiana, Roma, XII, VI, 1-2, pp. 273-276.

2003

- «Gourou Pierre », in Nouvelle Biographie Nationale, Académie Royale de Belgique, Bruxelles, 7, pp. 161-165.

- « Hegenscheidt Alfred », Nouvelle Biographie Nationale, Académie Royale de Belgique, Bruxelles, 7, pp. 191-193.

2004

- « Geography in Belgium », in VANDERMOTTEN C, NICOLAÏ H., MONTANARI A. (eds.), New trends and state of the geography in the European countries, Belgeo, 1, pp. 33-44.

- « In memoriam Roger-Ernest De Smet (1919-2004) », Belgeo, 2-3, pp. 195-197.

2005

- «L'image du Congo par la carte. De la boussole et de la planchette à l'avion ", in La mémoire du Congo. Le temps colonial, Africa Tervuren (Musée royal de l'Afrique centrale), Snoeck, pp. 139-149.

- « Le dernier avatar d'Elisée Reclus : le professeur d'université », in Elisée Reclus et nos géographies. Textes et prétextes, colloque international Lyon 7-9 septembre 2005, CD-Rom, 9 pages.

2006

- (avec J.M. Decroly), « Introduction », in DECROLY J.M. et NICOLAÏ H. (sous la direction de), Mutation des territoires dans le Monde à l'aube du XXI siècle, Coll. Géotextes,

L'Harmattan, Paris, pp. 7-11.

2007

- « La géographie, les géographes et le Congo », Mondes et Cultures, compte rendu annuel des travaux de l'Académie des Sciences d'Outre-Mer, Paris, LXVII, 1, pp. 337-349.

2008

- « Editorial », in Aspects of the History of the Belgian Geography and Cartography, Belgeo, 1, pp. 3-4.

- « Géographie et éducation esthétique. Les estampes scolaires de la Ville de Bruxelles », Belgeo, 1, pp. 47-68.

2009

- « Progrès de la connaissance du Congo, du Rwanda et du Burundi de 1993 à 2008 ", Belgeo, 3-4, pp. 247-404.

2012

- « Afriques Noires et Afriques ambiguës », EchoGéo, 20, http://echogeo.revues.org/13104;DOI : 10400/echogeo 13104

2013

- « Un guide colonial. Le guide du voyageur au Congo belge et au Ruanda-Urundi », Belgeo, 3, http://belgeo.revues.org/7161. 
- « Dossier. La vie villageoise dans le Kwango-Kwilu vers 1955 (1ère partie) », Belgeo, Dossiers et Archives, http://belgeo.revues.org/7357.

- « Dossier. La vie villageoise dans le Kwango-Kwilu vers 1955 (2e partie) », Belgeo, Dossiers et Archives, http://belgeo.revues.org/7360.

- « Le Congo et l'huile de palme. Un siècle. Un cycle ? ", Belgeo, 4, http:// belgeo.revues.org/11772.

\section{Collaboration aux Guides et itinéraires « Hommes et paysages » de la Société Royale Belge de Géographie}

-Itinéraire de l'habitat traditionnel en Famenne, 1990.

-Itinéraire de la frontière belgo-luxembourgeoise, 1999.

-Itinéraire de l'eau et de la laine en pays de Vesdre, 2002.

\section{Comptes rendus}

$S R B G$ : Bulletin de la Société Royale Belge de Géographie.

$R B G$ : Revue belge de Géographie.

SOBEG : Bulletin de la Société belge d'Études géographiques.

1959

- AUBERT de la RUE E., « L'homme et les Volcans », SRBG, 83, 3-4, p. 311.

- LASSERRE G., « Libreville », SRBG, 83, 3-4, pp. 303-307.

1960

- ANNAERT J., «Contribution à l'étude géographique de l'habitat et de l'habitation indigènes en milieu rural dans les Provinces orientale et du Kivu ", SRBG, 84, 2-4, , pp. 293-296.

1962

- CORNEVIN R., « Les Bassari du nord Togo », RBG, 86, 3, pp. 95-96.

- DESCHAMPS H., « Traditions orales et archives au Gabon. Contribution à l'ethnohistoire ", $R B G, 86,3$, pp. 97-08.

1963

- de PLANHOL X., « De la plaine pamphylienne aux lacs pisidiens », SOBEG, 32,

pp. 469-472.

1964

- CORNEVIN R., « Histoire du Congo (Léopoldville) », RBG, 88, 1-2, p. 187.

- DELVERT J., « Le paysan cambodgien », RBG, 88, 1-2, pp. 188-192.

1965

- LEBRUN J. et LEFEVRE P.C., « Enquête bibliographique sur la fertilité des sols et les éléments de sociologie rurale en Afrique au Sud du Sahara », SOBEG, 34, pp. 390-391.

1967

- BRUNET R., « Les campagnes toulousaines ; étude géographique », RBG, 91, 1-2-3, pp. 250-254.

- GEORGE P., « Sociologie et géographie », SOBEG, pp. 382-384.

1968

- LE ROY LADURIE E., « Histoire du climat depuis l'an mil », Revue Belge de Philologie et d'Histoire, 2, pp. 882-889.

- de PLANHOL X., «Les fondements géographiques de l'histoire de l'Islam », RBG, 92, 1-2, pp. 171- 176. 
- «Comité National de Géographie, Les géographes au service de la société », SOBEG, 38, pp. 142-144.

1972

- «Maghreb et Sahara. Etudes géographiques offertes à Jean Despois », RBG, 96, 2-3, pp. 247-248.

1973

- DENIS J., VENNETIER P. et WILMET J., « L'Afrique centrale et orientale », SOBEG, 42, pp. 207-209.

1974

- JOURET B., « La définition spatiale du phénomène urbain bruxellois », SOBEG, 43, pp. $165-167$.

1976

- KLATZMANN J., « Nourrir dix milliards d'hommes », RBG, 100, 2-3, pp. 333-334.

- PACKARD V., « Une société d'étrangers », RBG, 100, 2-3, pp. 365-366.

- SALMON P., « Histoire et critique », RBG, 100, 2-3, p. 337.

- SARFALVI B. (ed.), « Urbanization in Europe », RBG, 100, 2-3, p. 349.

1977

- HUETZ de LEMPS A., « L'Espagne », SOBEG, 46, pp. 110-112.

- PAYNE G.K., « Urban Housing in the Third World », RBG, 101, 1-3, pp. 233-234.

1978

- PICHERAL H., « Espace et Société. Géographie médicale du midi de la France », RBG, 102, 2-3, pp. 245-248.

1980

- KOLODNY E., « Les étrangers à Stuttgart », RBG, 104, 1, pp. 27-29.

1981

- WIESE B., « Zaire : Landesnatur, Bevölkerung, Wirtschaft », RBG, 105, 4, pp. 202-203.

1983

- ROTBERG R.I. \& RABB Th.K. (eds.), « Climate and History. Studies in Interdisciplinary History », Revue Belge de Philologie et d'Histoire, 61, 2, pp. 387-392.

- SMITH C.D. \& PARRY M. (eds.), « Consequences of Climatic Change », RBG, 107, 2, pp. 36-38.

- LAMB H.H., « Climate, History and the modern world », RBG, 107, 2, pp. 38-40.

- CLARK J.I. \& KOSINSKI L.A. (eds.), « Redistribution of Population in Africa », RBG, 107, 2, pp. 47-48.

- «Les Atlas des Départements et Territoires d'Outre-Mer : une série prestigieuse

(1975-1982) », RBG, 107, 6, pp. 168-169.

- SILVESTRE P. \& ARRAUDEAU M., « Le manioc », RBG, 107, 6, pp. 187-188.

- GOUROU P., « Terres de bonne espérance », RBG, 107, 6, pp. 183-186.

- PAIN M., « Kinshasa. Écologie et organisation urbaines », RBG, 107, 6, pp. 208-211.

- ROMLEY R.D.F. \& BROMLEY R., « South American Development : A Geographical Introduction », $R B G, 107,6$, p. 213.

- BONNENFANT P. (sous la direction de), « La péninsule arabique d'aujourd'hui, Tome II, Études par pays », RBG, 107, 6, pp. 214-215.

- MORISHIMA M (1982), « Why has Japan “succeeded” ? Western technology and Japanese ethos », RBG, 107, 6, p. 218.

1984 
- RAYNAUT C. (sous la direction de), « Milieu naturel, techniques, rapports sociaux ", RBG, 108, 3, pp. 184-185.

- UDO R.K., « The Human Geography of Tropical Africa », RBG, 108, 3, pp. 197-198.

1986

- JACQUOT M. \& COURTOIS B., « Le riz pluvial » ; ROUANET G., « Le maïs », RBG, 110, 4, p. 242.

- THUMERELLE P.J., «Peuples en mouvement. La mobilité spatiale des populations », $R B G, 110,4$, pp. 243-244.

- KLINGEBIEL A. \& MARQUETTE J.B. (sous la direction de), « La Grande Lande, Histoire naturelle et géographie historique », RBG, 110, 4, p. 253.

- O'CONNOR A., « The African City », RBG, 110, 4, pp. 258-260.

1988

- « Atlas d'Haïti », RBG, 112, 4, pp. 204-205.

- BROC N., « Dictionnaire illustré des explorateurs et grands voyageurs français du XIX ${ }^{e}$ siècle, I, Afrique ", RBG, 112, 4, p. 218.

1990

- DEMANGEOT J., « Les milieux "naturels" du globe », $3^{\mathrm{e}}$ éd., RBG, 114, 1, p. 17.

- « La ville et le fleuve, Ed. du Comité des travaux Historiques et scientifiques », RBG,

114, 1, pp. 17-18.

- SALMON P., « Le racisme devant l'histoire », $3^{e}$ éd., RBG, 114, 1, pp. 18-19.

- de PLANHOL X. (avec la collaboration de CLAVAL P.), « Géographie historique de la

France ", RBG, 114, 1, pp. 26-29.

- VENNETIER P. (sous la direction de), «La péri-urbanisation dans les pays tropicaux », RBG, 114, 1, pp. 33-35.

- GIRAUD Y. (éd.), « Le paysage de la Renaissance (Colloque de Cannes, 31 mai-2 juin 1985), Fribourg, Association d'Études sur l'Humanisme, la Réforme et la Renaissance ", Revue Belge de Philologie et d'Histoire, 73, 2, pp. 503-506.

1991

- BENOIT D., LEVANG P., PAIN M. (éditeur), SEVIN O., « Transmigration et migrations spontanées en Indonésie. Transmigration and spontaneous migrations in Indonesia »; PAIN M. (avec la collaboration de MUSHRITAH MUFTI, SRI DASWATI, NENG MARLINA), «Peuplement et développement régional en Indonésie, Settlement and regional development in Indonesia. Propinsi Lampung Sumatera Indonesia », ORSTOM, Departmen Transmigrasi ; « Peuplement et occupation de l'espace, Lampung Sumatera, 1905-1985 », RBG, 115, 4, pp. 366-370.

1993

- MORELLI A. (coordinatrice) (1991), « Ça ressemble à l'Italie. Spécificité de l'habitat italien en Wallonie et en Belgique », RBG, 117, 4, pp. 163-164.

- THUMERELLE P.J., « Atlas du Pas-de-Calais », RBG, 117, 4, pp. 166-168.

- BART F. (avec la collaboration de BART A.), « Montagnes d'Afrique, terres paysannes. Le cas du Rwanda », RBG, 117, 4, pp. 168-171.

- HUGON Ph. \& POURTIER R. (dir.), «Villes d'Afrique, Afrique contemporaine n` 168 », RBG, 117, 4, pp. 171-172.

- CHARRAS M. \& PAIN M. (éditeurs scientifiques), « Spontaneous settlements in Indonesia. Agricultural pioneers in Southern Sumatra. Migrations spontanées en Indonésie. La colonisation du sud de Sumatra », RBG, 117, 4, pp. 173-174. 1995 
- DENIS J. (éd.), « Géographie de la Belgique », Revue belge de Philologie et d'Histoire, 78, 4, pp. 1044-1051.

1996

- DEBIÉ F., « Géographie économique et humaine », RBG, 120, 4, pp. 292-293.

- VAN NAARSEN T., RUTTEN M., ZOOMERS A. (eds.), « The Diversity of Development. Essays in Honour of Jan Kleinpenning », RBG, 120, 4, pp. 293-294.

1998

- TAMISIER J.-C. (sous la direction de), « Dictionnaire des Peuples. Sociétés d'Afrique, d'Amérique, d'Asie et d'Océanie », RBG, 122, 1, pp. 85-86.

- « Bordeaux et l'Outre-Mer 1948-1997, Les Cahiers d'Outre-Mer, n 200 », RBG, 122, 1, pp. 87-89.

- TROCHET J.R., « La géographie historique de la France », RBG, 122, 1, pp. 92-93.

- KLEINPENNING J.M.G., « Peopling the Purple Land. A Historical Geography of Rural Uruguay 1500-1915 », RBG, 122, 1, pp. 98-100.

- de PLANHOL X., « Minorités en Islam. Géographie politique et sociale », RBG, 122, 1, pp. 101-103.

2002

- TAQUET Ph. (dir.), «Alcide d'Orbigny. Du Nouveau Monde... au passé du monde », Belgeo, 1, pp. 61-62.

- MONTANARI A. (ed.), « Food and Environment. Geographies of Taste », Belgeo, 1, pp. 62-63.

- HUETZ de LEMPS A., " Boissons et civilisations en Afrique », Belgeo, 1, pp. 66-67.

- POURTIER R. (2001), « Afriques noires », Belgeo, 1, pp. 68-69.

- RENARD J. (2002), « Les mutations des campagnes, Paysages et structures agraires dans le monde », Belgeo, 4, p. 390.

- CLAVAL P., « Géographie culturelle, une nouvelle approche des sociétés et des milieux », Belgeo, 4, pp. 393-394.

2004

- (avec S. Bastin), DUBRESSON A. \& RAISON J.P., «L'Afrique subsaharienne. Une géographie du changement », Belgeo, 4, pp. 503- 504.

- BART F., MBOLINE M.J. \& DEVENNE F. (dir.), « Kilimandjaro, montagne, mémoire, modernité », Belgeo, 4, pp. 505-506.

2006

- DIAMOND J., «Effondrement. Comment les sociétés décident de leur disparition ou de leur survie ", Belgeo, 4, pp. 493-497.

2008

- WILKINSON J.C., «A fatal duel. "Harry Alis" (1857-95), a behind scenes figure of the early Third Republic », Belgeo, 1, pp. 143-145.

- SALMON P., « Nouvelle introduction à l'histoire de l'Afrique », Belgeo, 1, pp. 145-146.

- VELLUT J.-L. (dir.), « Villes d'Afrique. Explorations en histoire urbaine », Belgeo, 1, pp. 147-149.

- VENNETIER P. (dir.), « À propos de développement rural en Afrique Noire », Belgeo, 1, pp. 149-154.

2009

- DUBOIS S., «La révolution géographique en Belgique. Départementalisation, administration et représentations du territoire de la fin du XVIII ${ }^{e}$ siècle au début du XIX siècle ", Belgeo, 2, pp. 234-237. 


\section{Divers}

Collaboration à Encyclopaedia Britannica, Encyclopaedia Universalis, Westermann Lexikon der Geographie, Bibliographie Géographique Internationale. 